\title{
Global existence of solutions for a fluid model of a neutron star
}

Jianlin Zhang*

\section{"Correspondence:}

mathzhangjianlin@hotmail.com

College of Information Science and

Technology, Donghua University,

Shanghai, 201620, P.R. China

Department of Applied

Mathematics, College of Science,

Zhongyuan University of

Technology, Zhengzhou, 450007,

P.R. China

\begin{abstract}
In this paper, we consider an initial-boundary value problem for the equations of a fluid spherical model of neutron star considered by Lattimer et al. We establish the global existence and regularity of the spherically symmetric solutions in $H^{i}(i=1,2,4)$ of this fluid model. These results improve and generalize the results of Ducomet and Necasova (Ann. Univ. Ferrara 55(1):153-193, 2009).
\end{abstract}

MSC: 35Q30; 35D30; 76N10

Keywords: neutron star; spherical case; global existence; regularity

\section{Introduction}

We consider an initial-boundary value problem for a fluid model of neutron star. In the case of a rapid cooling of the core of the star, the model used to describe the evolution of temperature in the star follows Lattimer et al. [2]. If a mechanical equilibrium is reached and the specific heat is a linear function of temperature, then the problem reduces to the study of a fast diffusion equation satisfied by the temperature in [3]. In a more general setting, suppose that the temperature is coupled to density and velocity fluctuations through a thermo-mechanical system; the simplest description of such a model is achieved through the compressible Navier-Stokes system in [4].

In this paper, we are interested in the 3D spherical symmetric solutions to the complete system, which has the general formulation as follows (see [5]):

$$
\left\{\begin{array}{l}
\rho_{t}+(\rho v)+\frac{2 \rho v}{r}=0 \\
\rho\left(v_{t}+v v_{r}\right)=\left(-p+\mu\left(v_{r}+\frac{2 v}{r}\right)\right)_{r}-4 v_{r} \frac{v}{r}+\rho F(r, t) \\
\rho\left(e_{t}+v e_{r}\right)=Q_{r}+\frac{2 Q}{r}-p\left(v_{r}+\frac{2 v}{r}\right)+\mu\left(v_{r}+\frac{2 v}{r}\right)^{2}-\frac{8 v v v_{r}}{r}-\frac{4 v v^{2}}{r^{2}}
\end{array}\right.
$$

in the domain $\omega \times \mathbb{R}^{+}$with $\omega:=\left(R_{0}, R_{1}\right)$, where $R_{0}$ is the radius of the internal rigid core of the star and $R_{1}$ is the exterior boundary, and $\rho(r, t)$ and $v(r, t)$ denote the density and the velocity, respectively. Let $\eta:=\frac{1}{\rho}$ be the specific volume and $\theta(r, t)$ be the temperature, then the pressure $p(\eta, \theta)=\frac{A}{2} \frac{\theta^{2}}{\eta^{2-\beta}}$ and the internal energy $e(\eta, \theta)=c_{\nu} \theta+\frac{A}{2(\beta-1)} \frac{\theta^{2}}{\eta^{1-\beta}}$, where constants $c_{v}>0, A>0$ and $1<\beta<2$. The heat flux $Q$ is given by the Fourier law $Q(\eta, \theta):=$

(c) 2016 Zhang. This article is distributed under the terms of the Creative Commons Attribution 4.0 International License (http://creativecommons.org/licenses/by/4.0/), which permits unrestricted use, distribution, and reproduction in any medium, provided you give appropriate credit to the original author(s) and the source, provide a link to the Creative Commons license, and indicate if changes were made. 
$\kappa(\eta, \theta) \theta_{r}$ with the following constraints on the thermal conductivity:

$$
\begin{aligned}
& \underline{\kappa}\left(1+\theta^{q}\right) \leq \kappa(\eta, \theta) \leq \bar{\kappa}\left(1+\theta^{q}\right), \\
& \left|\kappa_{\eta}(\eta, \theta)\right|+\left|\kappa_{\eta \eta}(\eta, \theta)\right| \leq \bar{K}_{1}\left(1+\theta^{q}\right), \\
& \left|\kappa_{\theta}(\eta, \theta)\right| \leq \bar{K}_{2}\left(1+\theta^{q-1}\right),
\end{aligned}
$$

for any $\theta \geq 0$, with positive constants $\underline{\kappa}, \bar{\kappa}, \bar{K}_{1}, \bar{K}_{2}$ and $q \geq 4 . F(r, t)$ is a given external field force (gravitation). Finally, we also assume the bulk viscous coefficient $\mu$ is a positive constant and the shear viscous coefficient $v=0$.

As in [1], we transform the system in Eulerian coordinates $(r, t)$ into that in Lagrangian (mass) coordinates $(x, t)$ by

$$
r(x, t):=r_{0}(x)+\int_{0}^{t} v(x, s) d s
$$

where $r_{0}(x):=\left(R_{0}^{3}+3 \int_{0}^{x} \eta(y, 0) d y\right)^{\frac{1}{3}}$ for $x \in(0, M)$, we have

$$
\left\{\begin{array}{l}
\eta_{t}=\left(r^{2} v\right)_{x} \\
v_{t}=r^{2}\left(-p+\mu \frac{\left(r^{2} v\right)_{x}}{\eta}\right)_{x}+f, \\
e_{t}=Q_{x}+\left(-p+\mu \frac{\left(r^{2} v\right)_{x}}{\eta}\right)\left(r^{2} v\right)_{x}, \\
r_{t}=v
\end{array}\right.
$$

in the fixed domain $\Omega \times \mathbb{R}^{+}$with $\Omega:=(0, M)$, where the specific volume $\eta$, the velocity $v$, the temperature $\theta$ and the radius $r$ depend on the Lagrangian mass coordinates. Now the heat flux is $Q(\eta, \theta)=\kappa(\eta, \theta) \frac{r^{4} \theta_{x}}{\eta}$ and the external field force is given by the Newtonian law $f(x)=-G \frac{M_{0}}{r^{2}}$, where $G$ and $M_{0}$ are positive constants. Denote the stress $\sigma$ by

$$
\sigma(\eta, \theta):=-p+\mu \frac{\left(r^{2} v\right)_{x}}{\eta}
$$

System (1.8)-(1.11) is subjected to the following boundary and initial conditions:

$$
\begin{aligned}
& \left.(\eta, v, r, \theta)\right|_{t=0}=\left(\eta_{0}, v_{0}, r_{0}, \theta_{0}\right)(x), \quad x \in[0, M], \\
& \left.v\right|_{x=0, M}=0,\left.\quad Q\right|_{x=0}=0,\left.\quad \theta\right|_{x=M}=\theta_{\Gamma}, \quad t \geq 0,
\end{aligned}
$$

with constant $\theta_{\Gamma}>0$.

Now let us recall some known results for the related system. For the full 3D compressible Navier-Stokes system with heat conductivity, we can refer to the basic references on the global existence of a weak solution, such as Lions [6], Feireisl [7], Feireisl and Novotný [8] and Bresch and Desjardins [9] and references therein. For the large-time behavior of the global solutions, we would also like to mention the work of Feireisl and Petzeltová $[10,11]$ and Feireisl and Novotný [12]. On the subject of the global existence and large-time behavior of smooth/strong solutions for the one-dimensional motions of viscous polytropic 
ideal gas under various conditions, we refer the reader to Kazhikhov and Shelukhin [13], Kawohl [14], Chen [15], Jiang [16-18], Zheng and Qin [19], Qin [20-22], and so on. For the free boundary problems, we can also refer to the work of Nagasawa [23, 24], Tani [25, 26] and Hsiao and Luo [27]. For the free and pure Neumann boundary value problem, we refer the reader to Umehara and Tani [28, 29], Qin and Huang [30], Qin et al. [31], and the references therein.

However, in the major part of astrophysical literature, for example, at least when rotation and magnetic aspects are neglected, a quite reliable approximation is spherical symmetry; see also [4, 32, 33]. In this quasi-monodimensional situation, the global existence and large-time behavior of a classical solution have been established in some spherically symmetric cases, and we refer to $[5,18,20,34-38]$ and the references therein. In addition, for the cylindrically symmetric Navier-Stokes equations with various boundary conditions, the global well-posedness of the solutions has been studied by many researchers, and we can refer to $[14,15,25,31,39-46]$ and the references therein. For problem (1.8)(1.13), Ducomet and Nečasová [1] have proved the global well-posedness and large-time asymptotics for the initial data $\left(\eta_{0}, v_{0}, \theta_{0}\right) \in H^{1} \times H^{1} \times H^{1}$. Ducomet and Nečasová [3] considered a fast diffusion equation satisfied by the temperature and proved well-posedness and large-time asymptotics of global solutions with the initial data $\theta_{0} \in L^{2}$.

In this paper, we shall establish the global existence and regularity of solutions for the spherical symmetric model of neutron star with the initial data $\left(\eta_{0}, v_{0}, \theta_{0}\right) \in H^{i} \times H^{i} \times$ $H^{i}(i=1,2,4)$. The main novelty is to establish the $H^{i}(i=1,2,4)$ regularity of the global solutions to problem (1.8)-(1.13). It is worth pointing out that the boundary condition on the temperature $\theta$ is different from general Dirichlet or Neumann boundary condition. Our results improve and generalize the results in [1].

In the following, the notations $L^{p}(1 \leq p \leq+\infty)$ and $W^{k, p}$ (in particular, $W^{k, 2}$ is also denoted by $H^{k}$ and $\left.H_{0}^{1}=W_{0}^{1,2}\right)$ stand for the usual Lebesgue spaces and the usual Sobolev spaces on $(0, M)$, respectively. $\|\cdot\|_{B}$ denotes the norm in the space $B,\|\cdot\|:=\|\cdot\|_{L^{2}} \cdot C^{\alpha, \beta}=$ $C^{\alpha, \beta}([0, M] \times[0, T])$ stands for uniformly Hölder continuous space with exponents $\alpha$ in $x$ and $\beta$ in $t$. We use $C_{0}$ and $C_{1}$ to denote a generic positive constant depending only on the parameters of the system and the bounds of the initial data $\left(\eta_{0}, v_{0}, \theta_{0}\right) \in\left(H^{1}([0, M])\right)^{3}$, but being independent of $t$. Furthermore, $C_{i}(T)(i=1,2,4)$ is a universal constant only dependent on the given time $T$, the physical constants and the initial data $\left(\eta_{0}, v_{0}, \theta_{0}\right) \in$ $\left(H^{i}([0, M])\right)^{3}$.

The rest of the paper is arranged as follows. In Section 2, we will state our main theorems about the global existence of the solutions to problem (1.8)-(1.13). Subsequently, by a series of lemmas, we shall prove our main theorems in Section 3.

\section{Main results}

Let $T$ be an arbitrary positive number. Now we give the definition of $H^{i}([0, M])$-solution to the initial-boundary problem (1.8)-(1.13).

Definition 2.1 Function $(\eta(x, t), v(x, t), \theta(x, t))$ is called a global $H^{i}([0, M])$-solution to problem (1.8)-(1.13) if it satisfies the following conditions:

$$
\begin{aligned}
& \eta(x, t) \in L^{2}\left([0, T], H^{i}([0, M])\right) \cap L^{\infty}\left([0, T], H^{i}([0, M])\right), \quad(x, t) \in[0, M] \times[0, T], \\
& v(x, t) \in L^{2}\left([0, T], H^{i+1}([0, M])\right) \cap L^{\infty}\left([0, T], H^{i}([0, M])\right), \quad(x, t) \in[0, M] \times[0, T],
\end{aligned}
$$


and

$$
\theta(x, t) \in L^{2}\left([0, T], H^{i+1}([0, M])\right) \cap L^{\infty}\left([0, T], H^{i}([0, M])\right), \quad(x, t) \in[0, M] \times[0, T],
$$

where $i=1,2,4$.

For convenience, we first state a proposition from [1].

Proposition 2.1 The corresponding static problem to problem (1.8)-(1.13) has a unique solution $(\bar{\eta}, \bar{\nu}, \bar{\theta})$ given by

$$
\left\{\begin{array}{l}
\bar{\eta}=\left[\frac{(\beta-1) G M_{0}}{(\beta-2) A \theta_{\Gamma}^{2}}\left(\frac{1}{r}-\frac{1}{r_{0}}\right)\right]^{-\frac{1}{\beta-1}}, \\
\bar{v}=0, \\
\bar{\theta}=\theta_{\Gamma},
\end{array}\right.
$$

where the constant $r_{0}$ only depends on the initial data.

We are now in a position to state our main result.

Theorem 2.1 Let the initial data $0<C_{0}^{-1}<\eta_{0}(x)<C_{0},\left(\eta_{0}, v_{0}, \theta_{0}\right) \in\left(H^{1}[0, M]\right)^{3}$. Assume that the heat conductivity $\kappa$ satisfies (1.4)-(1.6) and the initial data are compatible with boundary conditions. Then problem (1.8)-(1.13) admits a unique global $H^{1}([0, M])$-solution $(\eta(x, t), v(x, t), \theta(x, t))$ verifying, for all $(x, t) \in[0, M] \times[0, T]$,

$$
0<C_{1}^{-1} \leq \eta(x, t) \leq C_{1}, \quad 0<C_{1}^{-1} \leq \theta(x, t) \leq C_{1}, \quad 0<R_{0} \leq r(x, t) \leq R_{1},
$$

and

$$
\begin{aligned}
& \|\eta(t)-\bar{\eta}\|_{H^{1}}^{2}+\|v(t)\|_{H^{1}}^{2}+\|\theta(t)-\bar{\theta}\|_{H^{1}}^{2} \\
& \quad+\int_{0}^{t}\left(\|\eta-\bar{\eta}\|_{H^{1}}^{2}+\|v\|_{H^{2}}^{2}+\|\theta-\bar{\theta}\|_{H^{2}}^{2}+\left\|\eta_{t}\right\|^{2}+\left\|v_{t}\right\|^{2}+\left\|\theta_{t}\right\|^{2}\right)(s) d s \leq C_{1}(T) .
\end{aligned}
$$

Theorem 2.2 Let the initial data $0<C_{0}^{-1}<\eta_{0}(x)<C_{0},\left(\eta_{0}, v_{0}, \theta_{0}\right) \in\left(H^{2}[0, M]\right)^{3}$. Assume that the heat conductivity $\kappa$ satisfies (1.4)-(1.6) and the initial data are compatible with boundary conditions. Then problem (1.8)-(1.13) admits a unique global $H^{2}([0, M])$-solution $(\eta(x, t), v(x, t), \theta(x, t))$ verifying, for all $(x, t) \in[0, M] \times[0, T]$,

$$
\begin{aligned}
& \|\eta(t)-\bar{\eta}\|_{H^{2}}^{2}+\|v(t)\|_{H^{2}}^{2}+\|\theta(t)-\bar{\theta}\|_{H^{2}}^{2}+\left\|v_{t}(t)\right\|^{2}+\left\|\theta_{t}(t)\right\|^{2} \\
& \quad+\int_{0}^{t}\left(\|\eta-\bar{\eta}\|_{H^{2}}^{2}+\|v\|_{H^{3}}^{2}+\|\theta-\bar{\theta}\|_{H^{3}}^{2}+\left\|\eta_{t}\right\|_{H^{1}}^{2}+\left\|v_{t}\right\|_{H^{1}}^{2}+\left\|\theta_{t}\right\|_{H^{1}}^{2}\right)(s) d s \\
& \quad \leq C_{2}(T) .
\end{aligned}
$$

Theorem 2.3 Let the initial data $0<C_{0}^{-1}<\eta_{0}(x)<C_{0},\left(\eta_{0}, v_{0}, \theta_{0}\right) \in\left(H^{4}[0, M]\right)^{3}$. Assume that the heat conductivity $\kappa$ satisfies (1.4)-(1.6) and the initial data are compatible with 
boundary conditions. Then problem (1.8)-(1.13) admits a unique global $H^{4}([0, M])$-solution $(\eta(x, t), v(x, t), \theta(x, t))$ verifying, for all $(x, t) \in[0, M] \times[0, T]$,

$$
\begin{aligned}
& \|\eta(t)-\bar{\eta}\|_{H^{4}}^{2}+\|v(t)\|_{H^{4}}^{2}+\|\theta(t)-\bar{\theta}\|_{H^{4}}^{2}+\left\|\eta_{t}(t)\right\|_{H^{2}}^{2}+\left\|v_{t}(t)\right\|_{H^{2}}^{2}+\left\|\theta_{t}(t)\right\|_{H^{2}}^{2} \\
& \quad+\int_{0}^{t}\left(\|\eta-\bar{\eta}\|_{H^{4}}^{2}+\|v\|_{H^{5}}^{2}+\|\theta-\bar{\theta}\|_{H^{5}}^{2}+\left\|\eta_{t}\right\|_{H^{3}}^{2}+\left\|v_{t}\right\|_{H^{3}}^{2}+\left\|\theta_{t}\right\|_{H^{3}}^{2}+\left\|\eta_{t t}\right\|_{H^{1}}^{2}\right. \\
& \left.\quad+\left\|v_{t t}\right\|_{H^{1}}^{2}+\left\|\theta_{t t}\right\| w_{H^{1}}^{2}\right)(s) d s \leq C_{4}(T) .
\end{aligned}
$$

Corollary 2.1 Under the assumptions of Theorem 2.3 and some suitable compatibility conditions, the global solution $(\eta, v, \theta)$ to problem (1.8)-(1.13) is the classical solution verifying

$$
\|\eta\|_{C^{3, \frac{1}{2}}}+\|v\|_{C^{3, \frac{1}{2}}}+\|\theta\|_{C^{3, \frac{1}{2}}} \leq C_{4}(T) .
$$

Remark 2.1 The uniqueness of the global solutions has been obtained in [1].

Remark 2.2 Theorem 2.1 implies that problem (1.8)-(1.13) admits a unique global weak solution. Theorem 2.2 implies that problem (1.8)-(1.13) admits a unique global strong solution.

Remark 2.3 Our results generalize the previous work in [1].

\section{Proofs of theorems}

In this section, we will give some useful a priori estimates of the solutions to complete the proofs of the theorems.

\subsection{Global existence of $H^{1}$-solution}

In this subsection, we shall complete the proof of Theorem 2.1. As in [1], we have the following mass conservation and energy-entropy inequality.

Lemma 3.1 Under the assumptions in Theorem 2.1, the following estimates hold, for any $t \in[0, T]$,

$$
\begin{aligned}
& \int_{0}^{M} \eta(x, t) d x=\int_{0}^{M} \eta_{0}(x) d x, \\
& \int_{0}^{M}\left(\frac{1}{2} v^{2}+\frac{A}{2(\beta-1)} \eta^{\beta-1}\left(\theta-\theta_{\Gamma}\right)^{2}\right) d x \\
& \quad+\int_{0}^{t} \int_{0}^{M}\left(\frac{\kappa(\eta, \theta) r^{4}}{\eta \theta^{2}} \theta_{x}^{2}+\frac{\mu}{\eta \theta}\left(\left(r^{2} v\right)_{x}\right)^{2}\right) d x d s \leq C_{1} .
\end{aligned}
$$

Proof See, e.g., Lemma 1 in [1].

Lemma 3.2 Under the assumptions in Theorem 2.1, the following estimates hold for all $(x, t) \in \Omega \times[0, T]:$

$$
0<C_{1}^{-1} \leq \eta(x, t) \leq C_{1}, \quad 0<C_{1}^{-1} \leq \theta(x, t) \leq C_{1} .
$$


Proof See, e.g., Propositions 2 and 5 in [1].

Lemma 3.3 Under the assumptions in Theorem 2.1, the following estimate holds for any $t \in[0, T]:$

$$
\left\|\eta_{x}(t)\right\|^{2}+\left\|v_{x}(t)\right\|^{2}+\left\|\theta_{x}(t)\right\|^{2}+\int_{0}^{t}\left(\left\|\eta_{x}\right\|^{2}+\left\|v_{x x}\right\|^{2}+\left\|\theta_{t}\right\|^{2}\right)(s) d s \leq C_{1} .
$$

Proof See, e.g., Propositions 3-5 and Lemma 5 in [1].

Lemma 3.4 Under the assumptions in Theorem 2.1, the following estimate holds for any $t \in[0, T]:$

$$
\int_{0}^{t}\left(\left\|\theta_{x x}\right\|^{2}+\left\|v_{t}\right\|^{2}\right)(s) d s \leq C_{1}(T)
$$

Proof Multiplying (1.9) by $v_{t}$ over $(0, M) \times(0, T)$, employing an integration by parts and using Lemmas 3.1-3.3 and the Young inequality, we have

$$
\begin{aligned}
& \left\|\left(r^{2} v\right)_{x}\right\|^{2}+\int_{0}^{t}\left\|v_{t}(s)\right\|^{2} d s \\
& \leq C_{1}+C_{1} \int_{0}^{t} \int_{0}^{M}\left(\left|v_{t}\left(-r^{2} p_{x}+f\right)\right|+\left|\left(r^{2} v\right)_{x}\right|^{3}+\left|v v_{x}\right|\right) d x d s \\
& \leq C_{1}+\frac{1}{2} \int_{0}^{t}\left\|v_{t}(s)\right\|^{2} d s+C_{1} \int_{0}^{t} \int_{0}^{M}\left(\theta_{x}^{2}+\eta_{x}^{2}+f^{2}+v^{2}+v_{x}^{2}+\left|\left(r^{2} v\right)_{x}\right|^{3}\right) d x d s \\
& \leq C_{1}(T)+\frac{1}{2} \int_{0}^{t}\left\|v_{t}(s)\right\|^{2} d s+C_{1} \int_{0}^{t}\left\|\left(r^{2} v\right)_{x}\right\|_{L^{3}}^{3} d s \\
& \leq C_{1}(T)+\frac{1}{2} \int_{0}^{t}\left\|v_{t}(s)\right\|^{2} d s+C_{1} \int_{0}^{t}\left\|\left(r^{2} v\right)_{x}\right\|^{2}\left\|\left(r^{2} v\right)_{x x}\right\| d s \\
& \leq C_{1}(T)+\frac{1}{2} \int_{0}^{t}\left\|v_{t}(s)\right\|^{2} d s+C_{1} \int_{0}^{t}\left\|\left(r^{2} v\right)_{x x}\right\|^{2} d s \\
& \quad \leq C_{1}(T)+\frac{1}{2} \int_{0}^{t}\left\|v_{t}(s)\right\|^{2} d s,
\end{aligned}
$$

which implies

$$
\left\|\left(r^{2} v\right)_{x}\right\|^{2}+\int_{0}^{t}\left\|v_{t}(s)\right\|^{2} d s \leq C_{1}(T)
$$

Equation (1.10) can be rewritten as

$$
e_{\theta} \theta_{t}=Q_{x}-\theta p_{\theta}\left(r^{2} v\right)_{x}+\frac{\mu}{\eta}\left(r^{2} v\right)_{x}^{2}
$$

Multiplying (3.7) by $e_{\theta}^{-1} \theta_{x x}$, then integrating the result with respect to $x$ over $(0, M)$, using Hölder's inequality, the Sobolev embedding theorem, and Lemmas 3.1-3.3, we have, for 
any $\varepsilon>0$,

$$
\begin{aligned}
& \frac{d}{d t}\left\|\theta_{x}(t)\right\|^{2}+2 \int_{0}^{M} \frac{r^{4} \kappa}{e_{\theta} \eta} \theta_{x x}^{2} d x \\
& \quad=\int_{0}^{M}\left(\left(\frac{r^{4} \kappa}{\eta}\right)_{x} \theta_{x}-\theta p_{\theta}\left(r^{2} v\right)_{x}+\frac{\mu}{\eta}\left(r^{2} v\right)_{x}^{2}\right) \frac{\theta_{x x}}{e_{\theta}} d x \\
& \quad \leq \varepsilon\left\|\theta_{x x}\right\|^{2}+C_{1}(\varepsilon)\left(\left\|\theta_{x}\right\|^{2}+\left\|\eta_{x} \theta_{x}\right\|^{2}+\left\|\theta_{x}\right\|_{L^{4}}^{4}+\left\|v_{x}\right\|^{2}+\|v\|_{L^{4}}^{4}+\left\|v_{x}\right\|_{L^{4}}^{4}\right) \\
& \quad \leq \varepsilon\left\|\theta_{x x}\right\|^{2}+C_{1}(\varepsilon)\left(\left\|\theta_{x}\right\|^{2}+\left\|\theta_{x}\right\|_{L^{\infty}}^{2}+\left\|\theta_{x}\right\|^{3}\left\|\theta_{x x}\right\|+\left\|v_{x}\right\|^{2}+\|v\|^{3}\left\|v_{x}\right\|+\left\|v_{x}\right\|^{3}\left\|v_{x x}\right\|\right) \\
& \quad \leq 2 \varepsilon\left\|\theta_{x x}\right\|^{2}+C_{1}(\varepsilon)\left(\left\|\theta_{x}\right\|^{2}+\|v\|^{2}+\left\|v_{x}\right\|^{2}+\left\|v_{x x}\right\|^{2}\right) .
\end{aligned}
$$

Integrating (3.8) with respect to $t$ over $(0, t)$, taking $\varepsilon>0$ small enough, and using Lemmas 3.1 and 3.3 , we can obtain

$$
\left\|\theta_{x}(t)\right\|^{2}+\int_{0}^{t}\left\|\theta_{x x}(s)\right\|^{2} d s \leq C_{1}
$$

which, along with (3.6), leads to the estimate (3.5).

Now combining Lemmas 3.1-3.4 and noting equation (1.8), we complete the proof of Theorem 2.1.

\subsection{Global existence of $H^{2}$-solution}

In this subsection, we shall deal with the $H^{2}$-regularity of the global solutions to problem (1.8)-(1.13).

Lemma 3.5 Under the assumptions in Theorem 2.2, the following estimate holds for any $t \in[0, T]:$

$$
\left\|v_{x x}(t)\right\|^{2}+\left\|\theta_{x x}(t)\right\|^{2}+\left\|v_{t}(t)\right\|^{2}+\left\|\theta_{t}(t)\right\|^{2}+\int_{0}^{t}\left(\left\|v_{x t}\right\|^{2}+\left\|\theta_{x t}\right\|^{2}\right)(s) d s \leq C_{2}(T) .
$$

Proof See, e.g., Proposition 6 in [1].

Lemma 3.6 Under the assumptions in Theorem 2.2, the following estimate holds for any $t \in[0, T]:$

$$
\left\|\eta_{x x}(t)\right\|^{2}+\int_{0}^{t}\left\|\eta_{x x}(s)\right\|^{2} d s \leq C_{2}(T)
$$

Proof Differentiating (1.9) with respect to $x$, we have

$$
\begin{aligned}
\mu \frac{d}{d t}\left(\frac{\eta_{x x}}{\eta}\right)-p_{\eta} \eta_{x x}= & \left(r^{-2} v_{t}\right)_{x}+p_{\theta} \theta_{x x}+p_{\eta \eta} \eta_{x}^{2}+p_{\theta \theta} \theta_{x}^{2} \\
& +2 p_{\eta \theta} \eta_{x} \theta_{x}+2 \mu \frac{\eta_{x}}{\eta}\left(\frac{\left(r^{2} v\right)_{x}}{\eta}\right)_{x}-\left(r^{-2} f\right)_{x} \\
=: & \mathcal{M},
\end{aligned}
$$


where

$$
\|\mathcal{M}\| \leq C_{1}(T)\left(\left\|\theta_{x}\right\|_{H^{1}}+\left\|v_{t}\right\|+\left\|v_{x t}\right\|+\left\|\eta_{x}\right\|_{L^{4}}^{2}+\left\|v_{x}\right\|_{H^{1}}+1\right) .
$$

By Theorem 2.1 and Lemma 3.5, using Young's inequality, we get, for any $\varepsilon>0$,

$$
\int_{0}^{t}\|\mathcal{M}\|^{2} d s \leq C_{2}(T)+\varepsilon \int_{0}^{t}\left\|\eta_{x x}(s)\right\|^{2} d s
$$

Multiplying (3.12) by $\frac{\eta_{x x}}{\eta}$, then integrating the result over $[0, M] \times[0, t]$ and using Young's inequality and (3.13), taking $\varepsilon>0$ sufficiently small, we can obtain (3.11). Thus we complete the proof.

Lemma 3.7 Under the assumptions in Theorem 2.2, the following estimate holds for any $t \in[0, T]:$

$$
\int_{0}^{t}\left(\left\|v_{x x x}\right\|^{2}+\left\|\theta_{x x x}\right\|^{2}\right)(s) d s \leq C_{2}(T)
$$

Proof Differentiating (1.9) and (1.10) with respect to $x$, respectively, and using the Cauchy inequality, we easily obtain

$$
\left\|v_{x x x}(t)\right\| \leq C_{1}(T)\left(\left\|v_{x t}(t)\right\|+\left\|v_{x}(t)\right\|_{H^{1}}+\left\|\eta_{x}(t)\right\|_{H^{1}}+\left\|\theta_{x}(t)\right\|_{H^{1}}\right)
$$

and

$$
\left\|\theta_{x x x}(t)\right\| \leq C_{1}(T)\left(\left\|\theta_{x t}(t)\right\|+\left\|\theta_{x}(t)\right\|_{H^{1}}+\left\|\eta_{x}(t)\right\|_{H^{1}}+\left\|v_{x}(t)\right\|_{H^{1}}\right) .
$$

By virtue of Theorem 2.1 and Lemmas 3.5-3.6, we complete the proof.

Now combining Lemmas 3.5-3.7, we have completed the proof of Theorem 2.2.

\subsection{Global existence of $H^{4}$-solution}

In this subsection, we shall complete the proof of Theorem 2.3, which can be divided into the following lemmas.

Lemma 3.8 Under the assumptions of Theorem 2.3, we see that for any $t \in[0, T]$ and for $\varepsilon>0$ small enough,

$$
\begin{aligned}
&\left\|v_{x t}(x, 0)\right\|+\left\|\theta_{x t}(x, 0)\right\|+\left\|v_{t t}(x, 0)\right\|+\left\|\theta_{t t}(x, 0)\right\| \\
&+\left\|v_{t x x}(x, 0)\right\|+\left\|\theta_{t x x}(x, 0)\right\| \leq C_{4}(T) \\
&\left\|v_{t t}(t)\right\|^{2}+\int_{0}^{t}\left\|v_{t t x}(s)\right\|^{2} d s \leq C_{4}(T)+C_{2}(T) \int_{0}^{t}\left(\left\|\theta_{t x x}\right\|^{2}+\left\|v_{t x x}\right\|^{2}\right)(s) d s \\
&\left\|\theta_{t t}(t)\right\|^{2}+\int_{0}^{t}\left\|\theta_{t t x}(s)\right\|^{2} d s \leq C_{4}(T)+C_{2}(T) \varepsilon^{-1} \int_{0}^{t}\left\|\theta_{t x x}(s)\right\|^{2} d s \\
&+C_{1} \varepsilon \int_{0}^{t}\left(\left\|v_{t t x}\right\|^{2}+\left\|v_{t x x}\right\|^{2}\right)(s) d s .
\end{aligned}
$$


Proof Differentiating (1.9) and (1.10) with respect to $x$, respectively, using Theorems 2.1 and 2.2, we can get

$$
\begin{aligned}
\left\|v_{x t}(t)\right\| & \leq C_{2}(T)\left(\left\|v_{x}(t)\right\|_{H^{2}}+\left\|\theta_{x}(t)\right\|_{H^{1}}+\left\|\eta_{x}(t)\right\|_{H^{1}}+1\right), \\
\left\|\theta_{x t}(t)\right\| & \leq C_{2}(T)\left(\left\|\theta_{x}(t)\right\|_{H^{2}}+\left\|v_{x}(t)\right\|_{H^{1}}+\left\|\eta_{x}(t)\right\|_{H^{1}}+\left\|\theta_{x}(t)\right\|\right) .
\end{aligned}
$$

Similarly, differentiating (1.9) and (1.10) with respect to $x$ twice, respectively, we can infer from Theorems 2.1 and 2.2 that

$$
\begin{aligned}
\left\|v_{x x t}(t)\right\| \leq & C_{2}(T)\left(\left\|v_{x}(t)\right\|_{H^{3}}+\left\|\eta_{x}(t)\right\|_{H^{2}}+\left\|\theta_{x}(t)\right\|_{H^{2}}+\left\|v_{x}(t)\right\|_{L^{\infty}}\left\|\eta_{x x x}(t)\right\|\right. \\
& \left.+\left\|\eta_{x}(t)\right\|_{L^{\infty}}\left\|v_{x x x}(t)\right\|+\left\|v_{x x}(t)\right\|_{L^{\infty}}\left\|\eta_{x x}(t)\right\|+\left\|\eta_{x}(t)\right\|\right) \\
\leq & C_{2}(T)\left(\left\|v_{x}(t)\right\|_{H^{3}}+\left\|\eta_{x}(t)\right\|_{H^{2}}+\left\|\theta_{x}(t)\right\|_{H^{2}}\right), \\
\left\|\theta_{x x t}(t)\right\| \leq & C_{2}(T)\left(\left\|\theta_{x}(t)\right\|_{H^{3}}+\left\|\eta_{x}(t)\right\|_{H^{2}}+\left\|v_{x}(t)\right\|_{H^{2}}\right),
\end{aligned}
$$

or

$$
\begin{aligned}
\left\|v_{x x x x}(t)\right\| & \leq C_{2}(T)\left(\left\|v_{x}(t)\right\|_{H^{2}}+\left\|\eta_{x}(t)\right\|_{H^{2}}+\left\|\theta_{x}(t)\right\|_{H^{2}}+\left\|v_{t x x}(t)\right\|\right), \\
\left\|\theta_{x x x x}(t)\right\| & \leq C_{2}(T)\left(\left\|\theta_{x}(t)\right\|_{H^{2}}+\left\|\eta_{x}(t)\right\|_{H^{2}}+\left\|v_{x}(t)\right\|_{H^{2}}+\left\|\theta_{t x x}(t)\right\|\right) .
\end{aligned}
$$

It follows from (1.8) and (1.10) that

$$
\begin{aligned}
\left\|\eta_{t}(t)\right\| & \leq C_{1}\left(\|v(t)\|+\left\|v_{x}(t)\right\|\right), \\
\left\|\theta_{t}(t)\right\| & \leq C_{1}\left(\left\|\theta_{x x}(t)\right\|+\left\|\eta_{x}(t)\right\|+\left\|v_{x}(t)\right\|+\left\|v_{x x}(t)\right\|\right) .
\end{aligned}
$$

Differentiating (1.9) and (1.10) with respect to $t$, respectively, using Theorems 2.1-2.2 and (3.20)-(3.27), we have

$$
\begin{aligned}
\left\|v_{t t}(t)\right\| \leq & C_{2}(T)\left(\left\|v_{x}(t)\right\|_{H^{1}}+\left\|\eta_{x}(t)\right\|+\left\|\theta_{t}(t)\right\|\right. \\
& \left.+\left\|\theta_{x t}(t)\right\|+\left\|v_{t x}(t)\right\|+\left\|v_{t x x}(t)\right\|+\left\|\eta_{t}(t)\right\|\right) \\
\leq & C_{2}(T)\left(\left\|v_{x}(t)\right\|_{H^{3}}+\left\|\eta_{x}(t)\right\|_{H^{2}}+\left\|\theta_{x}(t)\right\|_{H^{2}}+1\right), \\
\left\|\theta_{t t}(t)\right\| \leq & C_{2}(T)\left(\left\|v_{x}(t)\right\|_{H^{1}}+\left\|\eta_{x}(t)\right\|+\left\|\theta_{t}(t)\right\|_{H^{2}}+\left\|\theta_{x}(t)\right\|_{H^{2}}+\left\|v_{t x}(t)\right\|\right) \\
\leq & C_{2}(T)\left(\left\|v_{x}(t)\right\|_{H^{2}}+\left\|\eta_{x}(t)\right\|_{H^{2}}+\left\|\theta_{x}(t)\right\|_{H^{3}}+1\right) .
\end{aligned}
$$

Thus the estimate (3.17) follows from (3.20)-(3.23), (3.29), and (3.31).

Differentiating (1.9) with respect to $t$ twice, multiplying the resultant by $v_{t t}$ and performing an integration by parts in $L^{2}(0, M)$, and using Theorem 2.2, the embedding theorem, and the Young inequality, we can derive

$$
\begin{aligned}
\frac{1}{2} \frac{d}{d t}\left\|v_{t t}\right\|^{2}= & -\int_{0}^{M}\left(r^{2} v_{t t}\right)_{x}\left(\mu \frac{\left(r^{2} v\right)_{x}}{\eta}-p\right)_{t t} d x-2 \int_{0}^{M}\left(\left(r^{2}\right)_{t} v_{t t}\right)_{x}\left(\mu \frac{\left(r^{2} v\right)_{x}}{\eta}-p\right)_{t} d x \\
& -\int_{0}^{M}\left(\left(r^{2}\right)_{t t} v_{t t}\right)_{x}\left(\mu \frac{\left(r^{2} v\right)_{x}}{\eta}-p\right) d x
\end{aligned}
$$




$$
\begin{aligned}
\leq & -\int_{0}^{M} \mu \frac{r_{x}^{4}}{\eta} v_{t t x}^{2} d x+C_{2}(T)\left(\left\|v_{t t}\right\|+\left\|v_{x t} v_{x}\right\|+\left\|v_{x}^{3}\right\|+\left\|\theta_{t} v_{x}\right\|\right. \\
& \left.+\left\|v_{x t}\right\|+\left\|\theta_{t t}\right\|+\left\|v_{x}^{2}\right\|\right)\left\|v_{t t x}\right\| \\
\leq & -C_{1}^{-1}\left\|v_{t t x}\right\|^{2}+C_{2}(T)\left(\left\|v_{x}\right\|_{H^{1}}^{2}+\left\|\theta_{t}\right\|^{2}+\left\|v_{x t}\right\|^{2}+\left\|\theta_{t t}\right\|^{2}+\left\|v_{t t}\right\|^{2}\right) .
\end{aligned}
$$

Thus, by Theorem 2.2,

$$
\left\|v_{t t}(t)\right\|^{2}+\int_{0}^{t}\left\|v_{t t x}(s)\right\|^{2} d s \leq C_{4}(T)+C_{2}(T) \int_{0}^{t}\left(\left\|v_{t t}\right\|^{2}+\left\|\theta_{t t}\right\|^{2}\right)(s) d s,
$$

which, together with (3.28) and (3.30), gives estimate (3.18).

Similarly, differentiating (1.10) with respect to $t$ twice, multiplying the result by $\theta_{t t}$ and performing an integration by parts over $L^{2}(0, M)$, and using the embedding theorem and the Young inequality, we have

$$
\begin{aligned}
\frac{1}{2} \frac{d}{d t} & \int_{0}^{M} e_{\theta} \theta_{t t}^{2} d x \\
= & -\int_{0}^{M}\left(\frac{r^{4} \kappa \theta_{x}}{\eta}\right)_{t t} \theta_{t t x} d x-\int_{0}^{M}\left(e_{\theta t t} \theta_{t}+e_{\eta t t}\left(r^{2} v\right)_{x}\right) \theta_{t t} d x-\frac{3}{2} \int_{0}^{M} e_{\theta t} \theta_{t t}^{2} d x \\
& -\int_{0}^{M}\left(e_{\eta}+p-\mu \frac{\left(r^{2} v\right)_{x}}{\eta}\right)\left(r^{2} v\right)_{x t t} \theta_{t t} d x+\int_{0}^{M}\left(\mu \frac{\left(r^{2} v\right)_{x}}{v}-p\right)_{t t}\left(r^{2} v\right)_{x} \theta_{t t} d x \\
& -2 \int_{0}^{M}\left(e_{\eta t}+\left(p-\mu \frac{\left(r^{2} v\right)_{x}}{\eta}\right)_{t}\right)\left(r^{2} v\right)_{x t} \theta_{t t} d x \\
= & : \sum_{i=1}^{6} P_{i} .
\end{aligned}
$$

By virtue of Theorems 2.1-2.2 and the embedding theorem, we deduce that, for any $\varepsilon \in$ $(0,1)$,

$$
\begin{aligned}
P_{1} \leq & -C_{1}\left\|\theta_{t t x}\right\|^{2}+C_{2}\left(\left\|\theta_{x}\right\|_{L^{\infty}}\left\|v_{x t}\right\|+\left\|v_{x}\right\|_{L^{\infty}}\left\|\theta_{x t}\right\|+\left\|v_{x}\right\|_{L^{\infty}}^{2}\left\|\theta_{x}\right\|\right. \\
& \left.+\left\|\theta_{x}\right\|_{L^{\infty}}\left\|\theta_{t}\right\|+\left\|\theta_{x}\right\|_{L^{\infty}}\left\|\theta_{t t}\right\|\right)\left\|\theta_{t t x}\right\| \\
\leq & -\left(2 C_{1}\right)^{-1}\left\|\theta_{t t x}\right\|^{2}+C_{2}(T)\left(\left\|\theta_{x t}\right\|^{2}+\left\|v_{x t}\right\|^{2}+\left\|v_{x}\right\|_{H^{1}}^{2}+\left\|\theta_{t t}\right\|^{2}\right), \\
P_{2} \leq & C_{1} \int_{0}^{M}\left(\left(\left|v_{x}\right|+\left|\theta_{t}\right|\right)^{2}+\left|v_{x t}\right|+\left|\theta_{t t}\right|\right)\left(\left|v_{x}\right|+\left|\theta_{t}\right|\right)\left|\theta_{t t}\right| d x \\
\leq & C_{1}\left\|\theta_{t t}\right\| \|_{L^{\infty}}\left(\left\|v_{x}\right\|+\left\|\theta_{t}\right\|\right)\left(\left(\left\|v_{x}\right\|_{L^{\infty}}+\left\|\theta_{t}\right\|_{L^{\infty}}\right)\left(\left\|v_{x}\right\|+\left\|\theta_{t}\right\|\right)+\left\|v_{x t}\right\|+\left\|\theta_{t t}\right\|\right) \\
\leq & C_{2}(T)\left(\left\|\theta_{t t}\right\|+\left\|\theta_{t t x}\right\|\right)\left(\left\|v_{x}\right\|_{H^{1}}+\left\|\theta_{t}\right\|+\left\|\theta_{x t}\right\|+\left\|v_{x t}\right\|+\left\|\theta_{t t}\right\|\right) \\
\leq & \varepsilon\left\|\theta_{t t x}\right\|^{2}+C_{2}(T) \varepsilon^{-1}\left(\left\|v_{x}\right\|_{H^{1}}^{2}+\left\|\theta_{t}\right\|^{2}+\left\|\theta_{x t}\right\|^{2}+\left\|v_{x t}\right\|^{2}+\left\|\theta_{t t}\right\|^{2}\right), \\
P_{3} \leq & C_{1} \int_{0}^{M}\left(\left|v_{x}\right|+\left|\theta_{t}\right|\right) \theta_{t t}^{2} d x \leq C_{1}\left\|\theta_{t t}\right\|_{L^{\infty}}\left(\left\|v_{x}\right\|+\left\|\theta_{t}\right\|\right)\left\|\theta_{t t}\right\| \\
\leq & C_{1}\left(\left\|\theta_{t t}\right\|+\left\|\theta_{t t x}\right\|\right)\left(\left\|v_{x}\right\|+\left\|\theta_{t}\right\|\right)\left\|\theta_{t t}\right\| \leq \varepsilon\left\|\theta_{t t x}\right\|^{2}+C_{2}(T) \varepsilon^{-1}\left\|\theta_{t t}\right\|^{2}, \\
P_{4} \leq & \varepsilon\left\|v_{t t x}\right\|^{2}+C_{2}(T) \varepsilon^{-1}\left\|\theta_{t t}\right\|^{2}, \\
P_{5} \leq & C_{2}(T)\left\|v_{x}\right\|_{L^{\infty}}\left\|\theta_{t t}\right\|\left(\left(\left\|v_{x}\right\|_{L^{\infty}}+\left\|\theta_{t}\right\|_{L^{\infty}}\right)\left(\left\|v_{x}\right\|+\left\|\theta_{t}\right\|\right)+\left\|v_{x t}\right\|\right.
\end{aligned}
$$




$$
\begin{aligned}
& \left.+\left\|\theta_{t t}\right\|+\left\|v_{x t t}\right\|+\left\|v_{t t}\right\|+\left\|v_{x}\right\|\right) \\
\leq & C_{2}(T)\left\|\theta_{t t}\right\|\left(\left\|v_{x}\right\|_{H^{1}}+\left\|\theta_{t}\right\|+\left\|\theta_{x t}\right\|+\left\|v_{x t}\right\|+\left\|\theta_{t t}\right\|+\left\|v_{x t t}\right\|+\left\|v_{t t}\right\|\right) \\
\leq & \varepsilon\left\|v_{t t x}\right\|^{2}+C_{2}(T) \varepsilon^{-1}\left(\left\|\theta_{t t}\right\|^{2}+\left\|v_{x}\right\|_{H^{1}}^{2}+\left\|\theta_{t}\right\|^{2}+\left\|\theta_{x t}\right\|^{2}+\left\|v_{x t}\right\|^{2}\right), \\
P_{6} \leq & C_{1} \int_{0}^{M}\left(\left|v_{x}\right|+\left|\theta_{t}\right|+\left|v_{x t}\right|+\left|v_{x}\right|^{2}+\left|v_{t}\right|\right)\left(\left|v_{x t}\right|+\left|v_{t}\right|\right)\left|\theta_{t t}\right| d x \\
\leq & C_{2}(T)\left\|v_{t x}\right\|^{\frac{1}{2}}\left\|v_{t x x}\right\|^{\frac{1}{2}}\left(\left\|v_{x}\right\|+\left\|\theta_{t}\right\|+\left\|v_{x t}\right\|\right)\left\|\theta_{t t}\right\|,
\end{aligned}
$$

which, by Hölder's inequality, implies

$$
\begin{aligned}
\int_{0}^{t} P_{6} d s \leq & C_{2}(T) \sup _{0 \leq s \leq t}\left\|\theta_{t t}(s)\right\|\left(\int_{0}^{t}\left\|v_{t x x}(s)\right\|^{2} d s\right)^{\frac{1}{4}}\left(\int_{0}^{t}\left\|v_{t x}(s)\right\|^{2} d s\right)^{\frac{1}{4}} \\
& \times\left(\int_{0}^{t}\left(\left\|v_{x}\right\|^{2}+\left\|\theta_{t}\right\|^{2}+\left\|v_{t x}\right\|^{2}\right)(s) d s\right)^{\frac{1}{2}} \\
\leq & \varepsilon\left(\sup _{0 \leq s \leq t}\left\|\theta_{t t}(s)\right\|^{2}+\int_{0}^{t}\|v t x x(s)\|^{2} d s\right)+C_{2}(T) \varepsilon^{-3}
\end{aligned}
$$

Thus it follows from (3.33)-(3.40) that, for any $\varepsilon \in(0,1)$ small enough,

$$
\begin{aligned}
& \left\|\theta_{t t}(t)\right\|^{2}+\int_{0}^{t}\left\|\theta_{t t x}(s)\right\|^{2} d s \\
& \leq C_{4}(T) \varepsilon^{-3}+C_{2}(T) \varepsilon^{-1} \int_{0}^{t}\left\|\theta_{t t}(s)\right\|^{2} d s \\
& \quad+C_{1} \varepsilon\left(\sup _{0 \leq s \leq t}\left\|\theta_{t t}(s)\right\|^{2}+\int_{0}^{t}\left(\left\|v_{t x x}\right\|^{2}+\left\|v_{t t x}\right\|^{2}\right)(s) d s\right) .
\end{aligned}
$$

Therefore taking the supremum in $t$ on the left-hand side of (3.41) and choosing $\varepsilon \in(0,1)$ small enough, we can derive estimate (3.19) from (3.30). The proof is complete.

Lemma 3.9 Under the assumptions of Theorem 2.3, the following estimates hold for any $t \in[0, T]$ and for $\varepsilon>0$ small enough:

$$
\begin{aligned}
& \left\|v_{x t}(t)\right\|^{2}+\int_{0}^{t}\left\|v_{x x t}(s)\right\|^{2} d s \leq C_{4}(T)+C_{2}(T) \varepsilon^{2} \int_{0}^{t}\left(\left\|v_{x t t}\right\|^{2}+\left\|\theta_{x x t}\right\|^{2}\right)(s) d s, \\
& \left\|\theta_{x t}(t)\right\|^{2}+\int_{0}^{t}\left\|\theta_{x x t}(s)\right\|^{2} d s \leq C_{4}(T)+C_{2}(T) \varepsilon^{2} \int_{0}^{t}\left(\left\|v_{x x t}\right\|^{2}+\left\|\theta_{x t t}\right\|^{2}\right)(s) d s .
\end{aligned}
$$

Proof Differentiating (1.9) with respect to $x$ and $t$, multiplying the result by $v_{x t}$ and integrating by parts in $L^{2}(0, M)$, we have

$$
\frac{1}{2} \frac{d}{d t}\left\|v_{x t}\right\|^{2}=N_{0}(t)+N_{1}(t)
$$

with

$$
N_{0}(t)=\left.\left(r^{2}\left(\mu \frac{\left(r^{2} v\right)_{x}}{v}-p\right)_{x}\right)_{t} v_{x t}\right|_{x=0} ^{x=L}, \quad N_{1}(t)=-\int_{0}^{M}\left(r^{2}\left(\mu \frac{\left(r^{2} v\right)_{x}}{v}-P\right)_{x}\right)_{t} v_{x x t} d x
$$


Using Theorem 2.2 and Lemma 3.8, the interpolation inequality, and Poincaré's inequality, we can get

$$
\begin{aligned}
N_{0}(t) \leq & C_{1}\left(\left(\left\|v_{x}\right\|_{L^{\infty}}+\left\|\theta_{x}\right\|_{L^{\infty}}\right)\left(\left\|v_{x}\right\|_{L^{\infty}}+\left\|\theta_{x}\right\|_{L^{\infty}}+\left\|\eta_{x}\right\|_{L^{\infty}}\right)+\left\|v_{x x}\right\|_{L^{\infty}}+\left\|\theta_{x t}\right\|_{L^{\infty}}\right. \\
& +\left\|v_{x x t}\right\|_{L^{\infty}}+\left\|\eta_{x}\right\|_{L^{\infty}}\left\|v_{x t}\right\|_{L^{\infty}}+\left\|v_{x}\right\|_{L^{\infty}}\left\|v_{x x}\right\|_{L^{\infty}}+\left\|v_{x}^{2}\right\|_{L^{\infty}}+\left\|\eta_{x t}\right\|_{L^{\infty}} \\
& +\left\|\eta_{x}\right\|_{L^{\infty}}\left\|\theta_{t}\right\|_{L^{\infty}}+\left\|v_{x}\right\|_{L^{\infty}}\left\|\theta_{x}\right\|_{L^{\infty}} \\
& \left.+\left\|\theta_{x}\right\|_{L^{\infty}}\left\|\theta_{t}\right\|_{L^{\infty}}+\left\|v_{x}\right\|_{L^{\infty}}\left\|\eta_{x}\right\|_{L^{\infty}}\right)\left\|v_{x t}\right\|_{L^{\infty}} \\
\leq & C_{2}(T)\left(N_{01}+N_{02}\right)\left\|v_{x t}\right\|^{\frac{1}{2}}\left\|v_{x x t}\right\|^{\frac{1}{2}}
\end{aligned}
$$

where

$$
N_{01}=\left\|v_{x}\right\|_{H^{2}}+\left\|\theta_{t}\right\|+\left\|\theta_{x t}\right\|
$$

and

$$
N_{02}=\left\|\theta_{x t}\right\|^{\frac{1}{2}}\left\|\theta_{x x t}\right\|^{\frac{1}{2}}+\left\|v_{x x t}\right\|^{\frac{1}{2}}\left\|v_{x x x t}\right\|^{\frac{1}{2}}+\left\|v_{x x t}\right\|+\left\|v_{x t}\right\|^{\frac{1}{2}}\left\|v_{x x t}\right\|^{\frac{1}{2}} .
$$

Applying Young's inequality several times, we have, for any $\varepsilon \in(0,1)$,

$$
\begin{aligned}
C_{2}(T) N_{01}\left\|v_{x t}\right\|^{\frac{1}{2}}\left\|v_{x x t}\right\|^{\frac{1}{2}} \leq & \frac{\varepsilon^{2}}{2}\left\|v_{x x t}\right\| w^{2} \\
& +C_{2}(T) \varepsilon^{-1}\left(\left\|v_{x}\right\|_{H^{2}}^{2}+\left\|\theta_{t}\right\|_{H^{1}}^{2}+\left\|v_{x t}\right\|^{2}\right)
\end{aligned}
$$

and

$$
\begin{aligned}
C_{2}(T) N_{02}\left\|v_{x t}\right\|^{\frac{1}{2}}\left\|v_{x x t}\right\|^{\frac{1}{2}} \leq & \frac{\varepsilon^{2}}{2}\left\|v_{x x t}\right\|^{2}+\varepsilon^{2}\left(\left\|\theta_{t x x}\right\|^{2}+\left\|v_{x x x t}\right\|^{2}\right) \\
& +C_{2}(T) \varepsilon^{-6}\left(\left\|\theta_{t x}\right\|^{2}+\left\|v_{x t}\right\|^{2}\right) .
\end{aligned}
$$

Thus it follows from (3.45)-(3.47) and Theorem 2.1 and Lemma 3.8 that

$$
\begin{aligned}
N_{0}(t) \leq & \varepsilon^{2}\left(\left\|v_{x x t}\right\|^{2}+\left\|\theta_{t x x}\right\|^{2}+\left\|v_{x x x t}\right\|^{2}\right) \\
& +C_{2}(T) \varepsilon^{-6}\left(\left\|\theta_{x}\right\|^{2}+\left\|v_{x}\right\|_{H^{2}}^{2}+\left\|\theta_{t x}\right\|^{2}+\left\|v_{x t}\right\|^{2}\right),
\end{aligned}
$$

which, along with Theorem 2.2, further yields

$$
\int_{0}^{t} N_{0}(s) d s \leq \varepsilon^{2} \int_{0}^{t}\left(\left\|v_{x x t}\right\|^{2}+\left\|\theta_{t x x}\right\|^{2}+\left\|v_{x x x t}\right\|^{2}\right)(s) d s+C_{2}(T) \varepsilon^{-6}
$$

Analogously, from Lemma 3.8, Theorem 2.1, and the embedding theorem, we can also derive that, for any $\varepsilon \in(0,1)$,

$$
\begin{aligned}
N_{1}(t) \leq & -\int_{0}^{M} \mu \frac{r^{4}}{\eta} v_{t x x}^{2} d x \\
& +C_{1}\left(\left(\left\|v_{x}\right\|+\left\|\theta_{t}\right\|+\left\|\eta_{x}\right\|\right)\left(\left\|\theta_{x}\right\|_{L^{\infty}}+\left\|\eta_{x}\right\|_{L^{\infty}}\right)+\left\|v_{x x}\right\|+\left\|\theta_{x t}\right\|\right.
\end{aligned}
$$




$$
\begin{aligned}
& \left.+\left\|\eta_{x}\right\|_{L^{\infty}}\left\|v_{x t}\right\|+\left\|v_{x}\right\|_{L^{\infty}}\left\|v_{x x}\right\|+\left\|v_{x}\right\|_{L^{\infty}}^{2}\left\|\eta_{x}\right\|+\left\|v_{x}\right\|+\left\|\theta_{x}\right\|+\left\|v_{x}\right\|^{2}\right)\left\|v_{x x t}\right\| \\
\leq & -\left(2 C_{1}\right)^{-1}\left\|v_{x x t}\right\|^{2}+C_{2}(T)\left(\left\|v_{x}\right\|_{H^{1}}^{2}+\left\|\theta_{t}\right\|_{H^{1}}^{2}+\left\|v_{x t}\right\|^{2}+\left\|\eta_{x}\right\|^{2}\right),
\end{aligned}
$$

which, combined with (3.44), (3.49), and Theorem 2.2 , shows that, for any $\varepsilon \in(0,1)$ small enough,

$$
\begin{aligned}
& \left\|v_{x t}(t)\right\|^{2}+\int_{0}^{t}\left\|v_{x x t}(s)\right\|^{2} d s \\
& \leq C_{2}(T) \varepsilon^{-6}+C_{1} \varepsilon^{2} \int_{0}^{t}\left(\left\|\theta_{t x x}\right\|^{2}+\left\|v_{x x x t}\right\|^{2}\right)(s) d s .
\end{aligned}
$$

On the other hand, differentiating (1.9) with respect to $x$ and $t$, we can derive from Theorem 2.2 and Lemma 3.8 that

$$
\begin{aligned}
\left\|v_{x x x t}(t)\right\| \leq & C_{1}\left\|v_{x t t}(t)\right\|+C_{2}(T)\left(\left\|v_{x}(t)\right\|_{H^{2}}+\left\|\theta_{x}(t)\right\|_{H^{1}}\right. \\
& \left.+\left\|\eta_{x}(t)\right\|_{H^{1}}+\left\|\theta_{t}(t)\right\|_{H^{2}}\right) .
\end{aligned}
$$

Thus inserting (3.52) into (3.51) leads to (3.42).

Similarly, by (1.10), we have

$$
\frac{1}{2} \frac{d}{d t} \int_{0}^{L} e_{\theta} \theta_{t x}^{2} d x=: \sum_{i=0}^{3} L_{i}(t)
$$

where

$$
\begin{aligned}
& L_{0}(t)=\left.\left(\frac{r^{4} \kappa \theta_{x}}{\eta}\right)_{x t} \theta_{x t}\right|_{x=0} ^{x=M}, \quad L_{1}(t)=-\int_{0}^{M}\left(\frac{r^{4} \kappa \theta_{x}}{\eta}\right)_{t x} \theta_{t x x} d x, \\
& L_{2}(t)=-\int_{0}^{M}\left(\left(e_{\eta}+p-\mu \frac{\left(r^{2} v\right)_{x}}{\eta}\right)\left(r^{2} v\right)_{x}\right)_{x t} \theta_{t x} d x, \\
& L_{3}(t)=-\int_{0}^{M}\left(e_{\theta t x} \theta_{t}+\frac{1}{2} e_{\theta t} \theta_{t x}+e_{\theta x} \theta_{t t}\right) \theta_{t x} d x .
\end{aligned}
$$

By virtue of the embedding theorem and the Young inequality, we derive from Lemmas 3.1, 3.8 , and (3.42) that, for any $\varepsilon \in(0,1)$,

$$
\begin{aligned}
L_{0}(t) \leq & C_{2}(T)\left(\left\|v_{x}\right\|_{H^{2}}+\left\|\theta_{x}\right\|_{H^{2}}+\left\|\theta_{t}\right\|_{H^{2}}+\left\|\theta_{x t}\right\|^{\frac{1}{2}}\left\|\theta_{x x t}\right\|^{\frac{1}{2}}\right. \\
& \left.+\left\|\theta_{x x t}\right\|^{\frac{1}{2}}\left\|\theta_{x x x t}\right\|^{\frac{1}{2}}\right)\left\|\theta_{x t}\right\|^{\frac{1}{2}}\left\|\theta_{x x t}\right\|^{\frac{1}{2}} \\
\leq & \varepsilon^{2}\left(\left\|\theta_{t x x}\right\|^{2}+\left\|\theta_{t x x x}\right\|^{2}\right)+C_{2}(T) \varepsilon^{-6}\left(\left\|v_{x}\right\|_{H^{2}}^{2}+\left\|\theta_{x}\right\|_{H^{2}}^{2}+\left\|\theta_{x t}\right\|^{2}\right), \\
L_{1}(t) \leq & -\left(2 C_{1}\right)^{-1}\left\|\theta_{t x x}\right\|^{2}+C_{2}(T)\left(\left\|v_{x}\right\|_{H^{1}}^{2}+\left\|\theta_{x}\right\|_{H^{1}}^{2}+\left\|\theta_{t}\right\|_{H^{1}}^{2}\right), \\
L_{2}(t) \leq & \varepsilon^{2}\left\|v_{t x x}\right\|^{2}+C_{2}(T) \varepsilon^{-2}\left(\left\|v_{x}\right\|_{H^{2}}^{2}+\left\|\theta_{t}\right\|_{H^{1}}^{2}+\left\|v_{x t}\right\|^{2}+\left\|\eta_{x}\right\|_{H^{1}}^{2}\right), \\
L_{3}(t) \leq & \varepsilon^{2}\left\|\theta_{t x x}\right\|^{2}+C_{2}(T) \varepsilon^{-2}\left(\left\|v_{x}\right\| w_{H^{1}}^{2}+\left\|\theta_{t}\right\|_{H^{1}}^{2}+\left\|\theta_{x}\right\|_{H^{2}}^{2}+\left\|v_{x t}\right\|^{2}+\left\|\eta_{x}\right\|^{2}\right) .
\end{aligned}
$$


Differentiating (1.10) with respect to $x$ and $t$, we can derive from Theorems 2.1-2.2 and Lemma 3.8 that

$$
\begin{aligned}
\left\|\theta_{t x x x}(t)\right\| \leq & C_{1}\left(\left\|\theta_{t t x}(t)\right\|+\left\|v_{x x t}(t)\right\|\right) \\
& +C_{2}(T)\left(\left\|v_{x}(t)\right\|_{H^{2}}+\left\|\eta_{x}(t)\right\|_{H^{2}}+\left\|\theta_{x}(t)\right\|_{H^{2}}+\left\|\theta_{x t}(t)\right\|\right) .
\end{aligned}
$$

Inserting (3.54)-(3.58) into (3.53) yields (3.43).

Lemma 3.10 Under the assumptions of Theorem 2.3, we have, for any $t \in[0, T]$,

$$
\begin{aligned}
& \left\|v_{t t}(t)\right\|^{2}+\left\|v_{x t}(t)\right\|^{2}+\left\|\theta_{t t}(t)\right\|^{2}+\left\|\theta_{x t}(t)\right\|^{2} \\
& \quad+\int_{0}^{t}\left(\left\|v_{t t x}\right\|^{2}+\left\|v_{x x t}\right\|^{2}+\left\|\theta_{t t x}\right\|^{2}+\left\|\theta_{x x t}\right\|^{2}\right)(s) d s \leq C_{4}(T), \\
& \left\|\eta_{x x x}(t)\right\|_{H^{1}}^{2}+\left\|v_{x x x}(t)\right\|_{H^{1}}^{2}+\left\|\theta_{x x x}(t)\right\|_{H^{1}}^{2}+\left\|v_{t x x}(t)\right\|^{2}+\left\|\theta_{t x x}(t)\right\|^{2} \\
& \quad+\int_{0}^{t}\left(\left\|v_{t t}\right\|^{2}+\left\|v_{x x t}\right\|_{H^{1}}^{2}+\left\|\theta_{t t}\right\|^{2}+\left\|\theta_{x x t}\right\|_{H^{1}}^{2}\right)(s) d s \leq C_{4}(T), \\
& \int_{0}^{t}\left(\left\|\eta_{x x x}\right\|_{H^{1}}^{2}+\left\|v_{x x x x}\right\|_{H^{1}}^{2}+\left\|\theta_{x x x x}\right\|_{H^{1}}^{2}\right)(s) d s \leq C_{4}(T) .
\end{aligned}
$$

Proof Adding (3.42)-(3.43) and choosing $\varepsilon>0$ small enough, we get

$$
\begin{gathered}
\left\|v_{x t}(t)\right\|^{2}+\left\|\theta_{x t}(t)\right\|^{2}+\int_{0}^{t}\left(\left\|v_{x x t}\right\|^{2}+\left\|\theta_{x x t}\right\|^{2}\right)(s) d s \\
\leq C_{4}(T)+C_{2}(T) \varepsilon^{2} \int_{0}^{t}\left(\left\|v_{x t t}\right\|^{2}+\left\|\theta_{x t t}\right\|^{2}\right)(s) d s .
\end{gathered}
$$

Now multiplying (3.18) and (3.19) by $\varepsilon$ and $\varepsilon^{\frac{3}{2}}$, respectively, then adding the results to (3.62) and taking $\varepsilon$ sufficiently small, we obtain (3.59).

Differentiating (1.9) with respect to $x$ and noting that $\eta_{x x t}=\left(r^{2} v\right)_{x x x}$, we get

$$
\mu \frac{\partial}{\partial t}\left(\frac{\eta_{x x}}{\eta}\right)-p_{\eta} \eta_{x x}=r^{-2} v_{t x}+K(x, t)-\left(r^{-2} f\right)_{x}-2 r^{-5} \eta v_{t}
$$

where

$$
\begin{aligned}
K(x, t)= & p_{\eta \eta} \eta_{x}^{2}+2 p_{\eta \theta} \theta_{x} \eta_{x}+p_{\theta \theta} \theta_{x}^{2}+p_{\theta} \theta_{x x}-2 \mu \frac{\eta_{x}^{2}}{\eta^{3}}\left(r^{2} v\right)_{x}+2 \mu \frac{\eta_{x}}{\eta^{2}}\left(r^{2} v\right)_{x x} \\
= & \frac{A(\beta-2)(\beta-3)}{2} \theta^{2} \eta^{\beta-4} \eta_{x}^{2}+2 A(\beta-2) \theta \eta^{\beta-3} \theta_{x} \eta_{x}+A \eta^{\beta-2} \theta_{x}^{2} \\
& +A \theta \eta^{\beta-2} \theta_{x x}+2 \mu\left(\frac{\eta_{x}}{\eta^{2}}\left(r^{2} v\right)_{x x}-\frac{\eta_{x}^{2}}{\eta^{3}}\left(r^{2} v\right)_{x}\right) .
\end{aligned}
$$

Differentiating (3.63) with respect to $x$, we have

$$
\mu \frac{\partial}{\partial t}\left(\frac{\eta_{x x x}}{\eta}\right)-p_{\eta} \eta_{x x x}=K_{1}(x, t),
$$


where

$$
\begin{aligned}
K_{1}(x, t)= & K_{x}(x, t)+p_{\eta x} \eta_{x x}+\mu\left(\frac{\eta_{x x} \eta_{x}}{\eta^{2}}\right)_{t}+r^{-2} v_{t x x}-4 r^{-5} \eta v_{t x} \\
& +10 r^{-8} \eta^{2} v_{t}-2 r^{-5} \eta_{x} v_{t}-\left(r^{-2} f\right)_{x x} .
\end{aligned}
$$

Obviously, it follows from Theorem 2.1 and Lemmas 3.8-3.9 that

$$
\left\|K_{1}(t)\right\| \leq C_{2}(T)\left(\left\|\eta_{x}(t)\right\|_{H^{1}}+\left\|v_{x}(t)\right\|_{H^{2}}+\left\|\theta_{x}(t)\right\|_{H^{2}}+\left\|v_{t x x}(t)\right\|\right)
$$

and

$$
\int_{0}^{t}\left\|K_{1}(s)\right\|^{2} d s \leq C_{4}(T)
$$

Multiplying (3.64) by $\frac{\eta_{x x x}}{\eta}$ over $L^{2}(0, M)$, we can obtain

$$
\frac{d}{d t}\left\|\frac{\eta_{x x x}}{\eta}\right\|^{2}+C_{1}^{-1}\left\|\frac{\eta_{x x x}}{\eta}\right\|^{2} \leq C_{1}\left\|K_{1}(t)\right\|^{2}
$$

which, along with (3.66), gives

$$
\left\|\eta_{x x x}(t)\right\|^{2}+\int_{0}^{t}\left\|\eta_{x x x}(s)\right\|^{2} d s \leq C_{4}(T) .
$$

It follows from (1.8)-(1.10) that

$$
\begin{aligned}
\left\|v_{x x x}(t)\right\| & \leq C_{2}(T)\left(\|v(t)\|_{H^{2}}+\left\|\eta_{x}(t)\right\|_{H^{1}}+\left\|\theta_{x}(t)\right\|_{H^{1}}+\left\|v_{x t}(t)\right\|\right), \\
\left\|\theta_{x x x}(t)\right\| & \leq C_{2}(T)\left(\|\theta(t)\|_{H^{2}}+\left\|\eta_{x}(t)\right\|_{H^{1}}+\left\|v_{x}(t)\right\|_{H^{1}}+\left\|\theta_{x t}(t)\right\|\right) .
\end{aligned}
$$

Using the embedding theorem, Theorems 2.1-2.2 and Lemmas 3.8-3.9, we can derive from (3.24)-(3.25), (3.59), and (3.68)-(3.70) that, for any $t \in[0, T]$,

$$
\begin{aligned}
& \left\|v_{x x x}(t)\right\|^{2}+\left\|\theta_{x x x}(t)\right\|^{2}+\left\|v_{x x}(t)\right\|_{L^{\infty}}^{2}+\left\|\theta_{x x}(t)\right\|_{L^{\infty}}^{2} \\
& \quad+\int_{0}^{t}\left(\left\|v_{x x x}\right\|_{H^{1}}^{2}+\left\|\theta_{x x x}\right\|_{H^{1}}^{2}+\left\|v_{x x}\right\|_{W^{1, \infty}}^{2}+\left\|\theta_{x x}\right\|_{W^{1, \infty}}^{2}\right)(s) d s \leq C_{4}(T) .
\end{aligned}
$$

Differentiating (1.9)-(1.10) with respect to $t$ and using Theorems 2.1-2.2 and Lemmas 3.83.9, we can deduce from (3.59), (3.68)-(3.71) that

$$
\begin{aligned}
\left\|v_{t x x}(t)\right\| \leq & C_{1}\left\|v_{t t}(t)\right\|+C_{2}(T)\left(\left\|v_{x}(t)\right\|_{H^{1}}+\left\|\eta_{x}(t)\right\|+\left\|\theta_{x}(t)\right\|\right. \\
& \left.+\left\|\theta_{t}(t)\right\|+\left\|\theta_{x t}(t)\right\|+\left\|v_{x t}(t)\right\|\right) \leq C_{4}(T), \\
\left\|\theta_{t x x}(t)\right\| \leq & C_{1}\left\|\theta_{t t}(t)\right\|+C_{2}(T)\left(\left\|v_{x}(t)\right\|_{H^{1}}+\left\|\eta_{x}(t)\right\|+\left\|\theta_{x}(t)\right\|_{H^{2}}\right. \\
& \left.+\left\|\theta_{t}(t)\right\|_{H^{1}}+\left\|v_{x t}(t)\right\|\right) \leq C_{4}(T),
\end{aligned}
$$


which, combined with (3.24)-(3.25) and (3.72), implies

$$
\begin{aligned}
& \left\|v_{x x x x}(t)\right\|^{2}+\left\|\theta_{x x x x}(t)\right\|^{2} \\
& \quad+\int_{0}^{t}\left(\left\|v_{t x x}\right\|^{2}+\left\|\theta_{t x x}\right\|^{2}+\left\|v_{x x x x}\right\|^{2}+\left\|\theta_{x x x x}\right\|^{2}\right)(s) d s \leq C_{4}(T) .
\end{aligned}
$$

Therefore it follows from (3.71), (3.74), and the embedding theorem that

$$
\left\|v_{x x x}(t)\right\|_{L^{\infty}}^{2}+\left\|\theta_{x x x}(t)\right\|_{L^{\infty}}^{2}+\int_{0}^{t}\left(\left\|v_{x x x}\right\|_{L^{\infty}}^{2}+\left\|\theta_{x x x}\right\|_{L^{\infty}}^{2}\right)(s) d s \leq C_{4}(T) .
$$

Now differentiating (3.64) with respect to $x$, we find

$$
\epsilon \frac{\partial}{\partial t}\left(\frac{\eta_{x x x x}}{\eta}\right)-p_{\eta} \eta_{x x x x}=K_{2}(x, t)
$$

where

$$
K_{2}(x, t)=K_{1 x}(x, t)+p_{\eta x} \eta_{x x x}+\mu\left(\frac{\eta_{x x x} \eta_{x}}{\eta^{2}}\right)_{t}
$$

From the embedding theorem and Lemmas 3.8-3.9 and (3.68)-(3.75), we can derive

$$
\begin{aligned}
\left\|K_{x x}(t)\right\| \leq & C_{4}(T)\left(\left\|v_{x}(t)\right\|_{H^{3}}+\left\|\theta_{x}(t)\right\|_{H^{3}}+\left\|\eta_{x}(t)\right\|_{H^{2}}\right), \\
\left\|K_{1 x}(t)\right\| \leq & C_{1}\left(\left\|K_{x x}(t)\right\|+\left\|v_{x x x t}\right\|+\left\|v_{x x t}\right\|+\left\|\left(p_{\eta x} \eta_{x x}\right)_{x}\right\|+\left\|\eta_{x} v_{x t}\right\|\right. \\
& \left.\quad+\left\|\eta_{x x}\right\|+\left\|\eta_{x} v_{t}\right\|+\left\|\eta_{x x} v_{t}\right\|+\left\|\left(\frac{\eta_{x} \eta_{x x}}{\eta^{2}}\right)_{x t}\right\|\right) \\
\leq & C_{1}\left\|v_{x x x t}(t)\right\|+C_{4}(T)\left(\left\|v_{x}(t)\right\|_{H^{3}}+\left\|\theta_{x}(t)\right\|_{H^{3}}+\left\|\eta_{x}(t)\right\|_{H^{2}}\right),
\end{aligned}
$$

whence

$$
\left\|K_{2}(t)\right\| \leq C_{1}\left\|v_{x x x t}(t)\right\|+C_{4}(T)\left(\left\|v_{x}(t)\right\|_{H^{3}}+\left\|\theta_{x}(t)\right\|_{H^{3}}+\left\|\eta_{x}(t)\right\|_{H^{2}}\right) .
$$

It follows from (3.28)-(3.31) that

$$
\int_{0}^{t}\left(\left\|v_{t t}\right\|^{2}+\left\|\theta_{t t}\right\|^{2}\right)(s) d s \leq C_{4}(T)
$$

which, along with (3.52) and (3.59), gives

$$
\int_{0}^{t}\left\|v_{x x x t}(s)\right\|^{2} d s \leq C_{4}(T)
$$

Thus from (3.68), (3.74), (3.77), and (3.79), it follows that

$$
\int_{0}^{t}\left\|K_{2}(s)\right\|^{2} d s \leq C_{4}(T)
$$


Multiplying (3.76) by $\frac{\eta_{x x x x}}{\eta}$ in $L^{2}(0, M)$, we can get

$$
\frac{d}{d t}\left\|\frac{\eta_{x x x x}}{\eta}\right\|^{2}+C_{1}^{-1}\left\|\frac{\eta_{x x x x}}{\eta}\right\|^{2} \leq C_{1}\left\|K_{2}(t)\right\|^{2}
$$

whence, by (3.80),

$$
\left\|\eta_{x x x x}(t)\right\|^{2}+\int_{0}^{t}\left\|\eta_{x x x x}(s)\right\|^{2} d s \leq C_{4}(T)
$$

Differentiating (1.10) with respect to $x$ and $t$, we can derive from Theorems 2.1-2.2 and Lemmas 3.8-3.9 that

$$
\left\|\theta_{t x x x}(t)\right\| \leq C_{1}\left\|\theta_{t t x}(t)\right\|+C_{2}(T)\left(\left\|v_{x}(t)\right\|_{H^{3}}+\left\|\eta_{x}(t)\right\|_{H^{2}}+\left\|\theta_{x}(t)\right\|_{H^{3}}+\left\|\theta_{x t}(t)\right\|\right) .
$$

Thus,

$$
\int_{0}^{t}\left\|\theta_{t x x x}(s)\right\|^{2} d s \leq C_{4}(T)
$$

Differentiating (1.9) with respect to $x$ three times, applying Lemmas 3.8-3.9, Theorems 2.12.2, and Poincaré's inequality, we have

$$
\left\|v_{x x x x x}(t)\right\| \leq C_{1}\left\|v_{t x x x}(t)\right\|+C_{2}(T)\left(\left\|v_{x}(t)\right\|_{H^{3}}+\left\|\eta_{x}(t)\right\|_{H^{3}}+\left\|\theta_{x}(t)\right\|_{H^{3}}\right) .
$$

Thus it follows from (3.74), (3.79), and (3.82) that

$$
\int_{0}^{t}\left\|v_{x x x x x}(s)\right\|^{2} d s \leq C_{4}(T)
$$

Similarly, we can differentiate (1.10) with respect to $x$ three times and use Lemmas 3.8-3.9, Theorems 2.1-2.2, Poincaré's inequality, (3.74), (3.82), and (3.84) to find

$$
\int_{0}^{t}\left\|\theta_{x x x x x}(s)\right\|^{2} d s \leq C_{4}(T)
$$

Hence, (3.60)-(3.61) follow from (3.74), (3.82), (3.86), and (3.87).

Finally, combining Lemmas 3.8-3.10, we complete the proof of Theorem 2.3.

\section{Competing interests}

The author declares that they have no competing interests.

\section{Acknowledgements}

The author would like to thank the referees for their valuable comments.

Received: 16 March 2016 Accepted: 15 June 2016 Published online: 24 June 2016

\section{References}

1. Ducomet, B, Nečasová, S: On a fluid model of neutron star. Ann. Univ. Ferrara 55(1), 153-193 (2009)

2. Lattimer, JM, Van Riper, KA, Prakash, M: Rapid cooling and the structure of neutron stars. Astrophys. J. 425, 802-813 (1994) 
3. Ducomet, B, Nečasová, S: Thermalization in a fluid model of neutron star. Discrete Contin. Dyn. Syst., Ser. B 3(3), 801-818 (2011)

4. Kippenhahn, R, Weingert, A: Stellar Structure and Evolution. Springer, Berlin (1994)

5. Fujita-Yashima, H, Benabidallah, R: Equation à symétris sphérique d'un gaz visqueux et calorifère avec la surface libre. Ann. Mat. Pura Appl. 168, 75-117 (1995)

6. Lions, PL: Mathematical Topics in Fluid Mechanics: Compressible Models. Oxford Lecture Series in Mathematics and Its Applications, vol. 2. Oxford University Press, Oxford (1996)

7. Feireisl, E: Dynamics of Viscous Compressible Fluids. Oxford University Press, Oxford (2004)

8. Feireisl, E, Novotný, A: Singular Limits in Thermodynamics of Viscous Fluids. Birkhäuser, Basel (2009)

9. Bresch, D, Desjardins, B: On the existence of global weak solutions to the Navier-Stokes equations for viscous compressible and heat conducting fluids. J. Math. Pures Appl. 87, 57-90 (2007)

10. Feireisl, E, Petzeltová, H: Large-time behaviour of solutions to the Navier-Stokes equations of compressible flow. Arch. Ration. Mech. Anal. 150, 77-96 (1999)

11. Feireisl, E, Petzeltová, H: On the long-time behavior of solutions to the Navier-Stokes-Fourier system with a time-dependent driving force. J. Dyn. Differ. Equ. 19, 685-707 (2007)

12. Feireisl, E, Novotný, A: Large time behaviour of flows of compressible, viscous, heat conducting fluids. Math. Methods Appl. Sci. 29(11), 1237-1260 (2006)

13. Kazhikhov, AV, Shelukhin, W: Unique global solution with respect to time of the initial-boundary value problems for one-dimensional equations of a viscous gas. J. Appl. Math. Mech. 41, 273-282 (1977)

14. Kawohl, B: Global existence of large solutions to initial boundary value problems for the equations of one-dimensional motion of viscous polytropic gases. J. Differ. Equ. 58, 76-103 (1985)

15. Chen, G: Global solution to the compressible Navier-Stokes equations for a reacting mixture. SIAM J. Math. Anal. 23 , 609-634 (1992)

16. Jiang, S: On initial boundary value problems for a viscous heat-conducting one-dimensional real gas. J. Differ. Equ. 110, 157-181 (1994)

17. Jiang, S: On the asymptotic behavior of the motion of a viscous, heat-conducting, one-dimensional real gas. Math. Z. 216(2), 317-336 (1994)

18. Jiang, S: Global spherically symmetric solutions of the equations of a viscous polytropic ideal gas in an exterior domain. Commun. Math. Phys. 178, 339-374 (1996)

19. Zheng, S, Qin, Y: Universal attractors for the Navier-Stokes equations of compressible and heat-conductive fluid in bounded annular domains in $R^{n}$. Arch. Ration. Mech. Anal. 160(2), 153-179 (2001)

20. Qin, Y: Nonlinear Parabolic-Hyperbolic Coupled Systems and Their Attractors. Advances in Partial Differential Equations, vol. 184. Birkhäuser, Basel (2008)

21. Qin, Y: Exponential stability for a nonlinear one-dimensional heat-conductive viscous real gas. J. Math. Anal. Appl. 272, 507-535 (2002)

22. Qin, Y: Universal attractor in $\mathrm{H}^{4}$ for the nonlinear one-dimensional compressible Navier-Stokes equations. J. Differ. Equ. 207, 21-72 (2004)

23. Nagasawa, T: On the outer pressure problem of the one-dimensional polytropic ideal gas. Jpn. J. Appl. Math. 5, 53-85 (1988)

24. Nagasawa, T: On the asymptotic behavior of the one-dimensional motion of the polytropic ideal gas with stress-free condition. Q. Appl. Math. 46(4), 665-679 (1988)

25. Tani, A: On the first initial-boundary value problem of compressible viscous fluid motion. Publ. Res. Inst. Math. Sci. 13, 193-253 (1977)

26. Tani, A: On the free boundary value problem for the compressible viscous fluid motion. J. Math. Kyoto Univ. $\mathbf{2 1}$ 839-859 (1981)

27. Hsiao, L, Luo, T: Large-time behaviour of solutions for the outer pressure problem of a viscous heat-conductive one dimensional real gas. Proc. R. Soc. Edinb., Sect. A, Math. 126(6), 1277-1296 (1996)

28. Umehara, M, Tani, A: Global solution to the one-dimensional equations for a self-gravitating viscous radiative and reactive gas. J. Differ. Equ. 234(2), 439-463 (2007)

29. Umehara, M, Tani, A: Temporally global solution to the equations for a spherically symmetric viscous radiative and reactive gas over the rigid core. Anal. Appl. 6, 183-211 (2008)

30. Qin, Y, Huang, L: Global Well-Posedness of Nonlinear Parabolic-Hyperbolic Coupled Systems. Frontiers in Mathematics. Springer, Basel (2012)

31. Qin, Y, Hu, G, Wang, T: Global smooth solutions for the compressible viscous and heat-conductive gas. Q. Appl. Math. 69(3), 509-528 (2011)

32. Chandrasekhar, S: An Introduction to the Study of Stellar Structures. Dover, New York (1967)

33. Chin, H-Y: Stellar Physics, Vol. I. Blaisdell, Waltham (1968)

34. Guo, Z, Li, H, Xin, Z: Lagrange structure and dynamics for solutions to the spherically symmetric compressible Navier-Stokes equations. Commun. Math. Phys. 309(2), 371-412 (2012)

35. Hoff, D: Spherically symmetric solutions of the Navier-Stokes equations for compressible, isothermal flow with large, discontinuous initial data. Indiana Univ. Math. J. 41(4), 1225-1302 (1992)

36. Jiang, S, Zhang, P: Global spherically symmetric solutions of the compressible isentropic Navier-Stokes equations, Commun. Math. Phys. 215, 559-581 (2001)

37. Nakamura, T, Nishibata, S, Yanagi, S: Large-time behavior of spherically symmetric solutions to an isentropic model of compressible viscous fluid in a field of potential forces. Math. Models Methods Appl. Sci. 14(12)، 1849-1879 (2004)

38. Qin, Y, Zhang, J, Su, X, Cao, J: Global existence and exponential stability of spherically symmetric solutions to the compressible combustion radiative and reactive gas. J. Math. Fluid Mech. (2016). doi:10.1007/s00021-015-0242-5

39. Chen, G, Hoff, D, Trivisa, K: Global solutions of the compressible Navier-Stokes equations with large discontinuous initial data. Commun. Partial Differ. Equ. 25, 2233-2257 (2000)

40. Chen, G, Hoff, D, Trivisa, K: Global solutions to a model for exothermically reacting, compressible flows with large discontinuous initial data. Arch. Ration. Mech. Anal. 166, 321-358 (2003)

41. Chen, G, Trivisa, K: Analysis on models for exothermically reacting, compressible flows with large discontinuous initial data. Contemp. Math. 371, 73-91 (2005) 
42. Ducomet, B: On the stability of a stellar structure in one dimension II: the reactive case. Math. Model. Numer. Anal. 31, 381-407 (1997)

43. Guo, B, Zhu, P: Asymptotic behavior of the solution to the system for a viscous reactive gas. J. Differ. Equ. 155, 177-202 (1999)

44. Qin, Y, Hu, G, Wang, T, Huang, L, Ma, Z: Remarks on global smooth solutions to a $1 \mathrm{D}$ self-gravitating viscous radiative and reactive gas. J. Math. Anal. Appl. 408(1), 19-26 (2013)

45. Qin, Y, Hu, G: Global smooth solutions for $1 \mathrm{D}$ thermally radiative magnetohydrodynamics. J. Math. Phys. 52, 023102 (2011)

46. Wang, D: Global solution for the mixture of real compressible reacting flows in combustion. Commun. Pure Appl. Anal. 3(4), 775-790 (2004)

Submit your manuscript to a SpringerOpen ${ }^{\odot}$ journal and benefit from:

- Convenient online submission

- Rigorous peer review

- Immediate publication on acceptance

- Open access: articles freely available online

- High visibility within the field

- Retaining the copyright to your article

Submit your next manuscript at $\boldsymbol{s p r i n g e r o p e n . c o m ~}$ 